\title{
Internal rotation of subdwarf B stars: limiting cases and asteroseismological consequences
}

\author{
Steven D. Kawaler ${ }^{1,2}$ \\ Shelbi R. Hostler 1,3
}

\begin{abstract}
Observations of the rotation rates of horizontal branch (HB) stars show puzzling systematics. In particular, cooler HB stars often show rapid rotation (with velocities in excess of $10 \mathrm{~km} / \mathrm{s}$ ), while hotter $\mathrm{HB}$ stars (those with $T_{\text {eff }}$ in excess of 11,000K) typically show much smaller rotation velocities of $8 \mathrm{~km} / \mathrm{s}$ or less. Simple models of angular momentum evolution of stars from the main sequence through the red giant branch fail to explain these effects. Assumption of solid body rotation throughout rotate much too slowly. On the other hand, assuming local conservation of angular momentum, but with solid body rotation in the convective regions, produces HB models also rotate too slowly at all $T_{\text {eff }}$ - but preserve a rapidly rotating core. In these cases, the observed angular velocities of HB stars require that some of the angular momentum stored in the core reaches the surface. Models that assume constant specific angular momentum in the surface convection zone have faster rotation in the envelope; while the predictions of these models match the observed rotation rates of the cooler HB stars, hot HB stars rotate more slowly than the models. Thus, while there is not as yet a coherent explanation of the trends of rotation on the HB, evolutionary models in all cases preserve a rapidly rotating core. To test the idea that HB stars contain such a core, one can appeal to detailed computations of trace element abundences and rotational mixing. However, a more direct probe is available to test these limiting cases of angular momentum evolution. Some of the hottest horizontal branch stars are members of the pulsating sdB class. They frequently show rich pulsation spectra characteristic of nonradially pulsating stars. Thus their pulsations probe the internal rotation of these stars, and should show the effects of rapid rotation in their cores. Using models of sdB stars that include angular momentum evolution, we explore this possibility and show that some of the sdB pulsators may indeed have rapidly rotating cores.
\end{abstract}

Subject headings: stars: rotation, stars: oscillations, stars: horizontal-branch, stars: variables: EC 14026 stars

\footnotetext{
${ }^{1}$ Department of Physics and Astronomy, Iowa State University, Ames, IA 50011 USA

2e-mail: sdk@iastate.edu

${ }^{3}$ current address: Submillimeter Array, 645 North A'ohoku Place Hilo, HI 96720
} 


\section{Introduction}

\subsection{Rotation of horizontal branch stars}

First studied in detail by R. Peterson in the mid 1980s (Peterson 1983, 1985a,b), the relativley rapid rotation of some horizontal branch stars remains an enigmatic feature of this phase of evolution. Rapid rotation was not seen in all horizontal branch (HB) stars, but the work by Peterson et al. (1995) on M13 suggested that rapid rotation was more common in cooler HB stars. Later observations by Behr et al. (2000a) of HB stars in M15 and in M13 (Behr et al. 2000b) further illuminate that there is a gap in the horizontal branches of M15 and M13, with two distinct rotation rates on either side. At effective temperatures less than about $11000 \mathrm{~K}$, HB stars frequently show rapid rotation $(v \sin i \approx 40 \mathrm{~km} / \mathrm{s}$ ) while no rotation rates above $10 \mathrm{~km} / \mathrm{s}$ appear in the hotter stars $\left(T_{\text {eff }}>11000 \mathrm{~K}\right)$. The work by Behr et al. $(2000 \mathrm{a}, \mathrm{b})$ agreed with a previous survey of M13 HB stars completed by Peterson et al. (1995). Further work by Behr (2003a) amplifies these results. In additon, a study of several other galactic globular clusters by Recio-Blanco et al. (2002) and Recio-Blanco et al. (2004) found similar results for the rotation of cool and hot HB stars. RR Lyra stars appear to show rotation with velocities below $10 \mathrm{~km} / \mathrm{s}$ (eg (Peterson et al. 1996)). While it is possible that the pulsations can drive a modest wind which in turn carries away angular momentum from these pulsating stars, this pause in the trend of rotation velocity with decreasing temperature is a mystery.

Age and metallicity are the primary factors in determining a cluster's features and placement in the $\mathrm{H}-\mathrm{R}$ diagram, and (in principle) the structure of the cluster's HB. Thus, if two clusters have similar metallicities and ages, one would expect them to show similar horizontal branch morphologies. But that is not the case; this is the infamous "second parameter problem" of globular cluster evolution. The rotation anomaly may couple to the other questions raised by HB morphology through the second parameter problem (Behr et al. 2000a,b; Behr 2003a) so an understanding of the rotational break could be important in understanding the fundamentals of stellar evolution.

Much of the work on horizontal branch stars naturally concentrates on stars in globular clusters. However, field stars that correspond to the post helium core flash of low-mass stars can perhaps play an important role in understanding the connection between rotation and HB morphology. For Population I stars, identification of post helium-flash objects is complicated by the paucity of stars in galactic clusters and distance determination for field stars. However, one class of such stars, the subdwarf B stars, are very hot $\left(T_{\text {eff }} \gtrsim 25,000 K\right.$ or more), and show high gravity $(\log g \approx 5.3-6.1)$. Thus they are readily identified as blue point sources in multicolor surveys, and spectroscopy reveals their stellar nature. They are the field analog to the bluest HB stars seen in clusters, and have very thin surface hydrogen layers.

But these stars present their own enigmas - in particular the origin of sdB stars. Their origin as stars which have lost most but not all of their hydrogen envelope following RGB evolution is mysterious, as simple models of RGB evolution cannot easily explain their miniscule surface 
hydrogen layers. Models proposed to explain their origin include interactions with a companion (eg. Sandquist et al. (2000); Mengel et al. (1976); Han et al. (2003))and even mass stripping by planetary companions (Soker \& Harpaz 2000). The high incidence of composite-spectrum binaries among the sdB stars is consistent with these kinds of models. Other scenarios involving singlestar evolution include variations of mass loss efficiency on the RGB (D'Cruz et al. 1996), and subsequently experience the core helium flash while on the helium-degenerate white dwarf cooling track (eg. Brown et al. (2001); Sweigart (1997)). In some cases, the proposed origin scenario makes a clear prediction about the rotational properties of sdB stars. For example, those that involve binary mergers or common envelope evolution should spin up the sdB product.

While there is no direct spectroscopic evidence because of the signature of chemical diffusion, the field sdB stars appear to be Population I objects. Their kinematics suggest that, in general, they derive from a younger population, and therefore we assume in our modeling that their progenitors have masses of $1 M_{\odot}$ or greater.

As far as their rotation, comparison with the results for Pop. II stars suggests that they all should be slow rotators, with $v \sin i \lesssim 10 \mathrm{~km} / \mathrm{s}$. Recently Behr (2003b) measured rotation velocities of a sample of field HB stars showed that a large majority are slow rotators, but that a sample of field HB stars do rotate faster. The work by Heber et al. (1999) has shown that at least one star, PG 1605, is a more rapid rotator - as expected based on an asteroseismic analysis of the pulsations by Kawaler (1998).

\subsection{Models of rotation of $\mathrm{HB}$ stars}

Attempts to understand the rotation observations, and perhaps link the rotation break to gaps in the horizontal branch or general HB morphology, illustrate the creativity of theorists. The lack of a coherent answer to date is a testament to the difficulty of the problem. Following up on a previous study of differential rotation in RGB stars (Pinsonneault et al. 1991), a detailed investigation by Sills \& Pinsonneault (2000) (hereafter SP2K) explored possible solutions based on single star evolution with different assumptions about internal angular momentum transport.

As we follow the same general procedure here in our study of sdB stars, we describe the computations of SP2K in some detail. SP2K modeled rotation on the red giant branch (RGB) and its effect on HB rotation. Starting with models that are rotating as solid bodies at the departure from the main sequence, SP2K followed evolution of the models through the helium flash and onto the HB. They studied several different limiting cases of angular momentum transport, two of which we follow up on in our study of Population I hot HB stars. Both cases conserve specific angular momentum (angular momentum per unit mass, $j$ ) in convectively stable regions. In convective regions, convective mixing is assumed to produce either constant angular velocity in the convection zone (Case B) or constant $j$ (Case D); in both cases, the angular momentum contained within the convection zone is preserved. These designations correspond to those used by SP2K. 
For low mass Population II stars, SP2K concluded that observations of the rapidly rotating cool HB stars are best understood with models with rapidly rotating cores that evolve from progenitors that have constant specific angular momentum in convection zones (i.e. Case D). Case D evolution produces models that can rotate at the rates seen in the cool HB stars. The slow rotation in the hotter HB stars requires some choking of angular momentum transport by chemical composition gradients produced by diffusion - such effects are seen in HB stars (and models) at temperatures above 11,000K (SP2K).

An alternative to SP2K's analysis is given by Soker \& Harpaz (2000), who investigated interaction with a planetary companion as one suggestion for a "second parameter." Soker \& Harpaz (2000) argued that the progenitors of the rapidly rotating HB stars have been spun up by planets or brown dwarfs, masses approximately 5 times Jupiter's mass and at separations of about 2 AU. While they state that these planetary interactions are the main second parameter, they also admit that planets do not completely solve the problem. The models of Soker \& Harpaz (2000) rely on horizontal branch angular momentum loss, not ineffective angular momentum transport as in SP2K to explain the slower rotating blue horizontal branch stars.

\subsection{Pulsating sdB stars - direct probes of internal rotation}

A subset of sdB stars are known to be multiperiodic pulsating stars. As nonradial modes must be present to explain the density of pulsation modes in a single star, we may be able to probe the interiors of these stars through asteroseismology. Such work on the EC 14026 stars (or sdBV stars), has already begun. The general properties of the sdBV stars are reviewed from an observational perspective by Kilkenny (2002); theoretical models of these stars are reviewed by Charpinet et al. (2001).

Models of rotation in single HB stars indicate that these stars should have rapidly rotating cores. Similarly, sdB stars that originate through binary mergers or common envelope evolution should show pathological rotation. The pulsating sdBV stars, if they represent "typical" sdB stars and, by extension, typical HB stars, should allow asteroseismology to address the state of internal rotation of HB stars.

In fact, one sdBV star, PG 1605 was found to have rapid rotation based on the asteroseismic analysis by Kawaler (1998) - this was later partially verified by Heber et al. (1999) through measurement of rotational broadening of spectral lines. Armed with this initial success, it is likely that asteroseismology can be a useful tool to explore important questions of the second parameter problem in globular clusters and the origins of sdB stars in the field. 


\subsection{This paper}

Most single-star models of HB stars that include rotation were developed for Pop II stars. The blue HB stars in globular clusters are quite old, and originate from stars that are less than $1 M_{\odot}$. Given the potential of sdBV stars for probing internal rotation, we therefore have computed new models of RGB stars and their hot HB decendents that are suited for seismic analysis appropriate to the sdBV stars.

The more recently formed single field HB stars such as the sdB stars probably originate from higher mass progenitors (up to the maximum mass that undergoes the core helium flash). This means that the stars that are now sdB stars could have had much higher initial angular momenta (Kawaler 1987) as well. Therefore, we have computed models of rotating RGB and sdB stars following the same strategy as Sills \& Pinsonneault (2000), but using higher mass Population I progenitors. Also, we compute a dense grid of models to adequately sample the various structures that might be relevant for the pulsators. We then look at the asteroseismic consequences of these rotation profiles, and begin to analyze the data on the pulsating sdB stars to see if they do show rapid core rotation.

This paper proceeds as follows. In the next section, we describe our stellar evolution code and our procedures for computing through the RGB and HB. We also discuss the limiting cases of angular momentum transport. Section 3 outlines the results of these calculations in terms of the surface rotation velocities and internal angular velocity profiles of the models. Using these profiles, we then use Section 4 to explore the influence of internal rotation on the observable pulsation frequencies in a preliminary asteroseismological analysis of the models. In Section 5 we discuss the results of the asteroseismological analysis in terms of the observed properties of the pulsators. We conclude in Section 6 with a discussion of our results, and an outline for more detailed study of the pulsators.

\section{Evolutionary Model Calculations}

\subsection{The evolution code and basic model parameters}

The evolution of our stellar models was computed using ISUEVO, the Iowa State University Evolution code. ISUEVO is a standard stellar evolution that is optimized for producing models for use in asteroseismology studies. ISUEVO computes models ranging from the ZAMS through the RGB and AGB. We also compute models of white dwarfs and planetary nebula central stars and of course the horizontal branch. Features of ISUEVO as applied to white dwarfs have been described in Kawaler (1993), Kawaler \& Bradley (1994), Dehner \& Kawaler (1995) and O'Brien \& Kawaler (2000), with further details in Dehner (1996). ISUEVO models have also been used for studies of HB stars (Stobie et al. 1997; van Hoolst et al. 1999; Kawaler 1998; Kilkenny et al. 1999; Reed et al. 2004) and AGB stars (Wood et al. 2004; Kawaler et al. 2003). 
The constitutive physics used in this code are described in the above references, but we summarize relevant details here. Opacities are taken from the OPAL compilation for standard mixtures (Iglesias \& Rogers 1996). Reaction rates utilize cross sections from Caughlan \& Fowler (1988). We follow the standard mixing length treatment with $\alpha=1.4$. During core helium burning on the HB we handle semiconvection in the core using a method similar to Castellani et al. (1985).

As we are modeling Population I stars, we assume a metallicity $\mathrm{Z}=0.02$ and an initial mixture that is solar. Model sequences were computed with initial masses of 1.0, 1.5, and $1.8 M_{\odot}$, and evolved from the ZAMS until degenerate ignition of helium in the core (the helium core flash). The core masses at the helium flash for these models are given in Table 1 (measured to the midpoint of the hydrogen-burning shell) along with a sample of others from the literature. For this paper, we did not compute the changes in the compositional structure caused by diffusion, and did not include mass loss while on the RGB. As dictated by observations, in the future we should probably consider models that deviate from this simple history; for example, in the case of RGB mass loss and late core helium flash such as computed by Brown et al. (2001), the core mass of hot horizontal branch stars could be significantly smaller than the standard values.

We did not follow the evolution of the model through the helium flash with our code, as the helium core flash may be a hydrodynamic event (Deupree 1996). Rather, we follow the conventional technique of computing a Zero Age Horizontal Branch (ZAHB) model that retains the properties of the RGB core at the flash (Sweigart \& Gross 1976). The hydrogen shell profile is fit to an analytic function during the construction of the ZAHB model. For envelopes thinner than $0.0003 M_{\odot}$, we had to reduce the thickness of the hydrogen to helium transition zone to preserve the surface abundance of hydrogen at the pre-flash value. For most models of interest for our study of sdB stars, the envelope thickness was too small to support an active hydrogen burning shell on the $\mathrm{ZAHB}$, and the stellar luminosity is derived entirely by the convective helium-burning core.

\subsection{Angular Momentum evolution}

The transport of angular momentum within stars that are evolving up the giant branch is dominated by the effects of convection in the deep convective envelope. Starting from some initial state on or just after the main sequence, the distribution of specific angular momentum $j$ would stay relatively constant, affected only by slow diffusive processes (and perhaps magnetic fields if present), in the absence of convection (Zahn 1992).

In the radiative regions of our models, we assume that $j$ is conserved locally, which is roughly equivalent to the shellular rotation described by Zahn (1992). We note here that the angular velocity profiles in our models represent the extreme case of no diffusion of $j$ and that, in real stars, the angular velocity profiles will be similar to or shallower than what we report. The total angular momentum within radiative zones is also an upper limit, as diffusive transport of $j$ usually drains angular momentum from the core into the more slowly spinning envelope. 
Within convection zones, we follow the procedure of SP2K and examine two limiting cases of $j$ transport. The first case, corresponding to SP2K's Case B, is that convective mixing produces constant angular velocity with radius in convection zones, with the total angular momentum of the convection zone being conserved. There is some observational evidence for this, for example helioseismic observations of the rotation of the solar convection zone show constant differential rotation with depth (but not necessarily with latitude) (Thompson et al. 1996). Case B models therefore transport $j$ outwards, as the outer layers of a convection zone have larger moments of inertia. For rapidly rotating convective regions (i.e. those where the rotation velocities are comparable to the speed of convective eddys) this seems like a reasonable approach.

The second case that we consider is comparable to SP2K, Case D: the total angular momentum within the envelope convection zone is conserved, and $j$ is constant within. In Case D evolution, then, the surface convection zone rotates faster in its inner regions where the radius (and therefore moment of inertia) is smaller. In essence, Case D treats $j$ as if it is a compositional element, mixing thoroughly throughout the convection zone. SP2K point out that such rotation in giant star envelopes is consistent with the much slower rotation velocities found in the convection zones of these stars. Even with our larger initial angular momenta (see below) the velocities of rotational motion are much smaller than typical convective velocities of a few $\mathrm{km} / \mathrm{s}$. This suggests that the small amount of differential rotation resulting from the assumption of constant $j$ can be maintained by the turbulent mixing generated by convection.

With the treatment of angular momentum described above, and since we do not include the effects of rotation on the hydrostatic structure on the models, we can treat the rotational evolution of the models as a "side calculation" during model evolution. In addition, we can compute the angular momentum distribution in a dimensionless way, by setting the initial model to constant angular velocity of 1 . Subsequent evolution results in changes in $\Omega$ and $j$ that are proportional to the initial value, allowing us to scale our results with a simple multiplicative factor that corresponds to the initial angular velocity (or, equivalently, the initial angular momentum).

For comparison with observations, we will generally assume that the initial rotation on the main sequence was as a solid body. The initial rotation rates for our models are taken from Kawaler $(1987)$ - for example, for the $1.8 M_{\odot}$ model, Kawaler $(1987)$ implies $v_{\text {rot }}=180 \mathrm{~km} / \mathrm{s}$, $J_{\text {init }}=3.17 \times 10^{50}$, and $\Omega=2.49 \times 10^{-5} s^{-1}$.

\subsubsection{Angular mometum transport time scales}

For this preliminary investigation, the only angular momentum transport we consider is what is required, ad hoc, to produce the two limiting cases described above. Other mechanisms must act as well - hydrodynamic and thermal instabilities that arise would all serve to transport angular momentum down the angular velocity gradients, effectively reducing the angular velocity contrasts that develop. These transport mechanisms will act on varying time scales. As long as these time 
scales are longer than or comparable to the evolutionary time scale on the RGB and HB, the real angular velocity profiles will resemble the limiting cases above. For reference, the shortest Eddington-Sweet circulation time scale within these models is approximately $10^{11}$ to $10^{12}$ years. However, given the steep gradients in $\Omega(r)$ that these models exhibit, other shear instabilities could act on much shorter time scales.

It is difficult to provide quantitative estimates of these time scales as they vary within the stellar model, and depend on unknown (and perhaps unknowable) properties such as the internal magnetic field strengths. However, for the purposes of this preliminary investigation, we are content to present and discuss the limiting case scenarios for the angular velocity under the two assumptions outlined above, with full acknowledgement that the real stars will not show such extreme behavior.

\section{Rotation rates and profiles}

Generally, our results for RGB rotation profiles are very similar to those of SP2K. In all cases, evolution up the RGB means an increase in radius (and moment of inertia) meaning that the outer layers slow down. At the same time, the core is contracting and therefor spinning up. Thus evolution up the RGB steepens the angular velocity profile. Figure 1 shows the evolution of the angular velocity during this phase for the $1.8 M_{\odot}$ Case B model (constant $\Omega$ in the surface convection zone). Since most of the moment of inertia is in the outermost layers, preserving the bulk angular momentum of the outer convection zone at a constant velocity means that the deepening (and expanding) star must spin down greatly. As shown in this figure, the surface layers rotate nearly 4 orders of magnitude more slowly than the core, reflecting the growth of the star by a factor of nearly 100 in radius.

The Case D model (constant $j$ through the surface convection zone) exhibits very similar behavior overall. However, the inner parts of the envelope have greater $j$ than the previous case (Case B), and must rotate faster with their smaller moment of inertia. Figure 2 shows the evolution of the angular velocity on the early RGB for the $1.8 M_{\odot}$ Case D model. In this case, as the convection zone deepens, the material that had a smaller $j$ is mixed with the envelope material with higher specific angular momentum, raising the angular velocity at the deepening base. For constant specific angular momentum, angular velocity decreases as moment of inertia increases, and the specific moment of inertia increases as radius squared. Thus the rotation rate increases with decreasing radius in such a convection zone. Material below the base of the convection zone has slowed by expansion, and an angular velocity kink develops just below the base of the envelope convection zone.

As shown by SP2K, the dominant feature of the internal rotation profile is the change in angular velocity that occurs near $0.27 M_{\odot}$ from the center of the model. The rotation profile drops

precipitously at this point as the result of draining of angular momentum from the core by the deepening, and then retreating, convective envelope. 
During early RGB evolution, the convective envelope appears and deepens. It reaches a maximum depth that is modestly dependent on the total stellar mass. The maxiumum depth that the surface convection zone reaches on the RGB is given in Table 1. Clearly, angular momentum transport in the convective envelope is critical in determining the eventual angular velocity profile of the model.

\subsection{HB rotation}

Following the helium core flash, the envelope is ejected, and the remnant core settles onto the horizontal branch. We have assumed that the specific angular momentum is preserved everywhere in the remnant core, and thus the angular velocity profile will reflect changes in the structure of the core as it converts from a degenerate configuration to a nondegenerate, helium burning region. As a degenerate core on the RGB, the structure resembles a polytrope with the same polytropic index as the convective helium burning core on the EHB (i.e. Hansen \& Kawaler (1994)). Therefore, the radius profile (as a function of mass) is a power law of the same power but different coefficient. The nondegenerate helium-burning core will have a larger radius, and therefore rotate more slowly (by the same factor) in the core than it did just prior to the helium core flash. Figure 3 shows this for the two cases of angular momentum transport in convection zones.

\subsubsection{Case B HB models}

Horizontal branch stars evolved from Case B RGB models show much smaller surface rotation velocities than those with Case $\mathrm{D}$ precursors. These models preserve a rapidly rotating core with roughly constant angular velocity out to about $0.3 M_{\odot}$. Material outside of that point had been drained of angular momentum by inclusion in the convective envelope early during RGB evolution, and so remain slowly rotating on the HB. With such small exterior rotation velocities, these stars contain a much larger contrast between the surface and core rotation velocities. Angular velocity drops by almost a factor of $10^{5}$, as shown in Figure 4 .

\subsubsection{Case D HB models}

The rotation profiles of models descended from Case D RGB evolution contain relics of the high specific angular momentum of material in the outer layers of the RGB progenitor.

When the constant $\mathrm{J} / \mathrm{M}$ envelope reached down to the $0.3 M_{\odot}$ zone, and then retreated, the higher specific angular momentum left behind produced a local maximum of angular velocity that persists into EHB evolution. The inner core never had a chance to share in this high angular momentum pool, and was left rotating more slowly. 
On the EHB, the core becomes convective, but does not reach the maximum angular velocity material. Therefore, the angular velocity profile changes little during EHB evolution - the core does spin down a bit as the angular momentum is mixed, but the inner core continues to spin about a factor of 5 slower than the fastest material. The angular velocity drops from the maximum by a factor of about 10 or so to the surface.

\subsection{Evolution of the angular velocity profile on the EHB}

The general features of the internal rotation profile remain nearly fixed through the stage of core helium burning. As shown in Figure 4, the rotation profile (with radius) shows the same general features at the start and end of HB evolution. For Case B evolution, a rapidly rotating core is preserved. For Case D evolution, the outer decrease in angular velocity is preserved, while the inner, more slowly rotating core, corresponding to the fully convective helium-burning zone, spins down at the start of EHB evolution as the core grows and mixes in lower angular momentum material. Further evolution results in little additional angular velocity change with radius. Given the uncertainty about the initial conditions (e.g. initial angular momentum on the main sequence, and main sequence angular momentum evolution), this relatively small change in the angular velocity profile on the EHB could allow efficient parameterization for studies of their seismic influence.

Given that our plan is to use these models for asteroseismology, the structure of the internal rotation profile is the focus of our study rather than the surface rotation velocities. Still, it is useful to examine the surface rotation rates of these models for general comparison with observation.

Given the way we construct HB models, the surface rotation velocity of models of different mass reflect the value of $j$ at the surface - which in turn results from the truncation of the envelope of the RGB progenitor. For Case B evolution, $j$ decreases with depth in the RGB progenitor. HB models with thicker envelopes therefore have surface layers with larger $j$, and faster rotation. However, since we are interested here in the bluest HB stars, the envelope masses are all less than $0.005 M_{\odot}$. The change in $j$ in the seed model over that small range is less than a factor of 2 . In the Case D models, $j$ is constant outside of the core - reflecting the assumptions built into the RGB model - so all of our Case D models have the same value of $j$ in their surface layers.

The surface angular velocity of the HB models depends on the initial value of $j$ at the surface (since $j \propto R^{2} \Omega$ ). The surface rotation velocity $v_{\text {rot }}$ scales as $R^{-1} \Omega$. Spectroscopic measurements of the sdB stars can determine $\log g\left(\propto R^{-2}\right)$ to adequate precision to compare with our models. Therefore, for a fixed $j$, the rotation velocity should scale with $g^{-1 / 2}$ with the constant of proportionality depending on the models and the initial angular momentum. The rotation velocities for sample models are shown in Figure 5 for a variety of initial rotation velocities. 


\section{Asteroseismology Probes}

The pulsating sdB stars - known as the EC 14026 stars or simply sdBV stars, are found in the midst of the $\left(\log g, T_{\text {eff }}\right)$ plane locus of the "ordinary" sdB stars. They range in $T_{\text {eff }}$ of slightly less than $30,000 \mathrm{~K}$ to over $35,000 \mathrm{~K}$, and have $\log g$ between 6.1 and 5.3. Most lie at the high-gravity end, clustering near the ZAEHB, with the cooler and lower gravity members numbering about $10 \%$ of the total. Figure 6 shows an H-R diagram that includes a representative sample of EC 14026 stars and sample evolutionary tracks from our sets of models. Given the convergence of the tracks in this plane, it is clear that $\mathrm{H}-\mathrm{R}$ diagram position alone is insufficient to judge their evolutionary state, with stars that are close to the ZAEHB intermixed with stars that have depleted helium in their cores.

The lowest gravity EC 14026 stars fall sufficiently far from the ZAEHB that they cannot be core helium-burners for core masses that are consistent with single-star evolution. Thus they are either on their way to the AGB or will evolve directly to the white dwarf cooling track in a very short time.

Another class of multiperiodic pulsating sdB stars exhibit longer periods (between 0.3 and 2.5 hours). These stars, colloquially known as the "Betsy stars" after their discoverer (Green et al. 2003), lie adjacent to the EC 14026 stars in the H-R diagram at the cool end. Fontaine et al. (2003) postulate that these are $g$-mode pulsators driven by the same mechanism that drives the pulsations in the EC 14026 stars.

The pulsators that lie in the main clump are shown in Figure 6 surrounded by a box that represents the mean values of $T_{\text {eff }}$ and $\log g$; the size of the box represents the standard deviation of these means. To explore the influence of a rapidly rotating core on the pulsations of sdBV stars, we employ representative models that fall within this box.

Many of the high-gravity pulsating sdB stars show complex pulsations that require a large number of independent periodicities. Examples include PG 1047 (Kilkenny et al. 2001) and PG 0014 (Brassard et al. 2001). The rich pulsation spectra show a mode density that is too high to be explained using only radial pulsations. Nonradially pulsating stars have a large number of available pulsation modes - manifested as a large number of possible oscillation frequencies. Even normal nonradial modes have a limited frequency distribution, meaning that some of the pulsators cannot be understood without resorting to either high values of $\ell$ (a large number of nodal lines on the stellar surface) or to rapid rotation.

Generally, for nonradial pulsations, each mode samples a slightly different part of the stellar interior, and so we can in principle use the observed pulsations to probe the structure of the stellar interior. Of particular relevance to this paper, nonradial modes are sensitive to stellar rotation rotation can significantly increase the number of distinct oscillation frequencies seen in pulsating stars by lifting the degenercy of modes with the same degree $(\ell)$ but different order $(m)$ as discussed below. 
In this section, we describe how nonradial pulsations in sdB stars may provide a window into their interiors, and test the hypothesis that horizontal branch stars have rapidly rotating interiors. The discussion here is intended to be somewhat general so as to illustrate the process by which we can detect rapid rotation of the stellar interior using currently observed sdBV stars. A future paper will describe our asteroseismic results in more detail and apply the analysis to individual pulsators.

\subsection{Rotational Splitting of Nonradial modes}

Nonradial pulsations in stars are generally expressed in terms of spheroidal modes. The spectrum of available oscillations are characterised by the eigenfrequency $\sigma_{n \ell m}$ corresponding to a spheroidal mode with quantum numbers $n, \ell$, and $m$. The values $\ell$ and $m$ refer to the spherical harmonic $Y_{\ell}^{m}$ with $\ell$ nodal lines, $m$ of which pass through the axis of symmetry. The sign of $m$ gives the direction of propogation of the corresponding surface running wave, and therefore there are $2 \ell+1$ possible values of $m$ for a given $\ell$. The radial order of the mode (corresponding roughly to the number of nodes in the radial direction) is $n$.

For perfect spherical symmetry, the oscillation frequencies are independent of $m$ and depend only on $n$ and $\ell$. However, if rotation (or another non-spherical process) is present, the value of the oscillation frequency will depend on $m$. In the case of slow rotation as a solid body, the $m$ dependence of the oscillation frequency can be written as:

$$
\sigma_{n \ell m}=\sigma_{n \ell 0}+m \Omega\left(1-C_{n \ell}\right)
$$

where the quantity $C_{n, \ell}$ is a function of the spatial structure of the oscillations within the star (the eigenfunctions). For the above case of slow rotation,

$$
C_{n \ell}=\frac{\int_{0}^{R}\left(2 \xi_{r} \xi_{h}+\xi_{h}^{2}\right) \rho r^{2} d r}{\int_{0}^{R}\left(\xi_{r}^{2}+\ell[\ell+1] \xi_{h}^{2}\right) \rho r^{2} d r}
$$

where the radial and horizontal perturbations at $r$ are given by $\xi_{r}$ and $\xi_{h}$ respectively. For a more complete description of rotational splitting, see one of the standard texts of stellar pulsation (i.e. Cox (1980); Unno et al. (1989)).

Equations (1) and (2) correspond to solid body rotation with a rotation frequency that is small compared to the oscillation frequency. The constancy of $\Omega$ means that it does not appear inside the integral in Equation (2), allowing simple determination of the effect of rotation in this special case. Clearly, rotation can split an $\ell=1$ oscillation into a triplet, spaced in frequency by the rotation frequency times $\left(1-C_{n \ell}\right)$. Sequences of such equally spaced triplets have been clearly identified in a number of pulsating white dwarf stars, for example, suggesting that they rotate nearly as solid bodies, at least in those portions of those stars that contribute to the integral (2) (Kawaler et al. 1999). 
If a star undergoes differential rotation with radius - that is, if $\Omega=\Omega(r)$, then the simple form for calculating the value of $\sigma_{n \ell m}$ above is not appropriate. The splitting in this case (where $\Omega$ depends on radius, not on latitude) is given by

$$
\sigma_{n \ell m}=\sigma_{n \ell 0}+m \int_{0}^{R} \Omega(r) K_{n \ell}(r) d r
$$

where

$$
K_{n \ell}=\frac{\left(\xi_{r}^{2}-[\ell(\ell+1)-1] \xi_{h}^{2}-2 \xi_{r} \xi_{h}\right) \rho r^{2}}{\int_{0}^{R}\left(\xi_{r}^{2}+\ell[\ell+1] \xi_{h}^{2}\right) \rho r^{2} d r}
$$

is the rotation kernel corresponding to a mode with $n$ and $\ell$.

Thus for a star with internal differential rotation with radius, the observed splitting for a mode with a given $n$ and $\ell$ represents an average of $\Omega(r)$ weighted by the rotation kernel $K_{n \ell}(r)$. If the kernel function of a mode is nonzero where the star has very rapid rotation, then the resulting splitting can reveal the inner rapid rotation despite slow rotation at the surface layers.

\subsection{Sample rotation kernels and results for $\mathrm{sdB}$ models}

Figure 7 shows sample rotation kernels for an sdB model representative of the pulsating EC 14026 stars. This particular model is nearing the end of core helium burning as a middle-aged sdBV model. The modes illustrated span the period range seen in the pulsating sdBV stars. Note that higher $n$ modes have more peaks, but that in all modes the kernel has nonzero value close to the stellar core. Also indicated in Figure 7 are lines representing $\log \Omega(r)$ for Case B angular

momentum transport in the RGB outer convection zone. Note that even though the amplitude of $K_{n \ell}$ is small in the inner regions, the large value of $\log \Omega(r)$ in the same region suggests that the value of the rotational splitting will be dominated by the core rotation.

The case for $\ell=2$ modes is more striking. For several modes in this model, the rotation kernel is quite large in the core. This likely results from resonant mode trapping by the composition transition zones, in particular the $\mathrm{He} / \mathrm{C}+\mathrm{O}$ zone in the core. At this evolutionary stage, the model shows some mixed-character modes for $\ell=2$ which can have significant amplitude in the core (Charpinet et al. 2002).

Tables 2-7 present the computed splittings for models of different evolutionary stages that lie within the box of Figure 6. The first two tables give periods and splittings for a near-ZAHB model; Tables 4 and 5 show the same range of periods in a middle-aged sdB model, and Tables 5 and 6 show results for a highly evolved sdB model. Tables show the splittings expected if the model is rotating as a solid body at the surface rate, and for models with differential rotation. The splittings for differential rotation in models with Case B are given in Tables 2, 4, and 6; Case D models are in Tables 3, 5, and 7.

As an illustration of the kind of effects that differential rotation can produce, consider Tables 
4 and 5, which present the computed splittings for two models of middle-aged sdB stars. In the Case B model in Table 4, the surface rotation velocity, if representative of the entire star, would produce a splitting of less than $0.01 \mu \mathrm{Hz}$ for all modes (surface rotation velocity of only $6 \mathrm{~m} / \mathrm{s}$ ), yet the rapid internal rotation results in splittings ranging from 1 to $80 \mu \mathrm{Hz}$ depending on the value of $n$ and $\ell$ for the mode.

Table 5 shows what happens for a Case D model, which has an artificially scaled-down rotation rate of $10 \mathrm{~km} / \mathrm{s}$ at the surface (this model would have been rotating way too quickly if it had the initial angular momentum assigned from the main sequence progenitor). For this model, the expected splitting would be about $15 \mu \mathrm{Hz}$ given the surface rate and the assumption of solid body rotation. The rapidly rotating core, however, produces splittings ranging from 24 to $52 \mu \mathrm{Hz}$.

In both limits, the rotational splitting is much larger than expected from assuming solid body rotation at the surface rotation velocity. Even more interesting is the fact that the splitting changes (sometimes dramatically) from one mode to the next within a sequence of the same $\ell$ but different $n$. This reflects the fact that the low-order modes in these stars sample portions of the core and envelope, and the exact parts sampled depend on the overtone number. With a steep drop in $\Omega$, the kernel functions (see Figure 7 as an example) sweep across these fluctuations as $n$ increases. In addition, mode trapping by the composition zones present in these models produces some strong core localization of the kernels for certain modes, and these therefore show larger rotational splitting.

The fact that the expected splittings can be quite large, coupled with the large differences in expected splittings from mode to mode, can make observational identification of rotational splitting quite difficult. Generally, the way this has been done in the past is to look for a sequence of equallyspaced triplets in a Fourier transform, and then identify them as $\ell=1$ for example. Even if all members of such triplets are not seen, finding several pairs of modes split by the same amount (or twice that amount, or half) would be indicative of rotational splitting. For sdBV stars, though, with significant mode-to-mode differences in splittings expected, a series of rotationally split frequencies would be virtually indistinguishable from modes of different $\ell$ and $n$. For example, consider the model of an advanced-stage sdB star whose periods are listed in Tables 6 and 7 . The $\ell=2$ modes have periods that are close to $\ell=0$ (radial) modes. With splittings of 40 to $200 \mu \mathrm{Hz}$ for the lowest order modes, the quintuplets $\ell=2, n=0,2$ and 3 could spread over very large frequency ranges that would encompass, or nearly reach, $\ell=0, n=1 ; \ell=0, n=2$; and $\ell=1, n=3$. Without appealing to differential rotation, such a rich mode spectrum would require appealing to other mechanisms to explain the periodicities.

\section{Conclusions}

We have shown that sdB stars, like horizontal branch stars in general, should retain rapidly rotating cores as a relic of their evolution on the RGB. Such rapid rotation could serve as a reservoir of angular momentum which, when tapped, can produce anomalously fast rotation at the surface 
on the HB. Taken alone, our results are consistent with the models of SP2K in that without late angular momentum transport, standard models of single HB stars cannot explain observed trends in the distribution of surface rotation velocities with effective temperature. However, if angular momentum transport can tap the internal reservoir in the absence of composition gradients, the suggestion of SP2K that redder HB stars can spin up in the latter stages of core helium burning remains theoretically viable.

Other mechanisms can be at work that would serve to reduce or eliminate the strong differential rotation present in these limiting-case models. Also, other scenarios for the origin of sdB stars (such as binary mergers) and of sdB surface rotation velocities (i.e. spin-up by nearby planets) make specific predictions about the internal angular velocity profiles. In any case, though, we can test the predictions of a wide variety of stellar models if we can measure the rotation rate of the subsurface layers of HB stars and, in particular, the hot subdwarfs.

Pulsations can probe the interiors of these stars and thereby reveal the rotation velocity profile within. Rapidly rotating cores produce rotational splitting of nonradial modes, and the amount of the splittings is not what would be expected from spectroscopic measurements of $v \sin i$. Evidence that we see such effects could be the rotation of PG 1605 as deduced from the pulsations. The rotatonal splitting identified in that star by Kawaler (1998) is about 3 times larger than the $v \sin i$ measured via spectroscopy (Heber et al. 1999). While this could simply be the result of a small inclination angle between the rotation axis of the star and our line of sight, it could also result from differential rotation and a rapidly rotating core.

Clearly, it is important to consider the possibly large rotational splittings that might appear in the pulsation spectra of the EC 14026 stars. Identification of modes with large splittings, or of sequences of modes with varying splitting would point towards strong differential rotation. In either case, such a measurement would confirm the existence of a rapidly rotating core within these stars. Failure to find such effects would be convincing evidence that angular momentum transport mechanisms on the RGB or during the HB phase are very efficient at wiping out angular velocity

gradients. Should that be the case, then the mystery of the rotation of horizontal branch stars will remain with us.

This work is partially supported by NSF Grant AST20205983 and by the NASA Astrophysics Theory Program through grant NAG-58352, both to Iowa State University.

\section{REFERENCES}

Behr, B.B., Cohen, J.G., \& McCarthy, J.K. 2000a, ApJ, 531, L37

Behr, B.B., Djorgovski, S.G., Cohen, J.G., McCarthy, J.K., Cote, P., Piotto, G., \& Zoccali, M. 2000b, ApJ, 528, 849 
Behr, B.B. 2003a, ApJS, 149, 67

Behr, B.B. 2003b, ApJS, 149, 101

Brassard, P., Fontaine, G., Billères, M., Charpinet, S., Liebert, J., \& Saffer, R. 2001, ApJ, 563, 1013

Brown, T.M., Sweigart, A.V., Lanz, T., Landsman, W.B., \& Hubeny, I. 2001, ApJ, 562, 368

Castellani, V., Chieffi, A., Pulone, L., \& Tornambé, A. 1985, ApJ, 296, 204

Caughlan, G.R. \& Fowler, W.A. 1988, Atomic Data Nuc. Data Tables, 40, 284

Charpinet, S., Fontaine, G., \& Brassard, P. 2001, PASP, 113, 775

Charpinet, S., Fontaine, G., Brassard, P., \& Dorman, B. 2002, ApJS, 139, 487

Cox, J.P. 1980, Stellar Pulsation (Princeton: Princeton University Press)

D’Cruz, N., Dorman, B., Rood, R., \& O’Connell, R. 1996, ApJ, 466, 359

Dehner, B.T. \& Kawaler, S.D. 1995, ApJ, 445, L141

Dehner, B.T. 1996, Ph.D. dissertation, Iowa State University

Deupree, R. 1996, ApJ, 471, 377

Green, E.M. et al. 2003, ApJ, 583, L31

Han, Z., Podsiadlowski, P., Maxsted, P., \& Marsh, T. 2003, MNRAS, 341, 669

Hansen, C.J. \& Kawaler, S.D. 1994, Stellar Interiors: Principles, Structure, and Evolution, (New York: Springer)

Heber, U., Reid, I.N., \& Werner, K. 1999, å, 348, L25

Iglesias, C.A. \& Rogers, F.J. 1996, ApJ, 464, 943

Kawaler, S.D. 1987, PASP, 99, 1322

Kawaler, S.D. 1993, ApJ, 404, 294

Kawaler, S.D. \& Bradley, P.A. 1994, ApJ, 427, 415

Kawaler, S.D. 1998, in 11th European Workshop on White Dwarfs, ed. J.-E. Solheim \& E. Meistas, (San Francisco: ASP), 158.

Kawaler, S.D., Sekii, T., \& Gough, D. 1999, ApJ, 516, 349 
Kawaler, S.D., Hostler, S.R., \& Burkett, J. 2003, in NATO Advanced Research Workshop on White Dwarfs: the Thirteenth European Workshop, ed. R. Silvotti \& D. de Martino (Kluwer: Dordrecht)

Kilkenny, D., Koen, C., O’Donoghue, D., Van Wyk, F., Larson, K.A., Shobbrook, B., Sullivan, D.J., Burleigh, M., Dobbie, P., \& Kawaler, S.D. 1999, MNRAS, 303, 525

Kilkenny, D., Billères, M., Stobie, R.S., Fontaine, G., Shobbrook, R.R., O’Donoghue, D., Brassard, P., Sullivan, D., Burleigh, M.R., \& Barstow, M.A. 2002, MNRAS, 331, 399

Kilkenny, D. 2002, in Radial and Nonradial Pulsations as Probes of Stellar Physics, ed. C. Aerts \& T. Bedding (San Francisco, ASP Press), p. 356

Mengel, J.G., Norris, J., \& Gross, P. 1976, ApJ, 204, 488

O’Brien, M.S. \& Kawaler, S.D. 2000, ApJ, 539, 372

Peterson, R.C. 1983, ApJ, 275, 537

Peterson, R.C. 1985a, ApJ, 289, 320

Peterson, R.C. 1985b, ApJ, 294, L35

Peterson, R.C., Rood, R.T., \& Crocker, D.A. 1995, ApJ, 453, 214

Peterson, R.C., Carney, B.W., \& Lathank D.W. 1996, ApJ, 465, L47

Pinsonneault, M. H., Deliyannis, C. P., \& Demarque, P. 1991, ApJ, 367, 239

Recio-Blanco, A., Piotto, G., Aparicio, A., \& Renzini, A. 2002, ApJ, 572, L71

Recio-Blanco, A., Piotto, G., Aparicio, A., \& Renzini, A. 2004, A\&A, 417, 597

Reed, M. et al. (the WET collaboration) 2004, MNRAS, 348, 1164

Sandquist, E., Taam, R., \& Burkert, A. 2000, ApJ, 533, 984

Sills, A. \& Pinsonneault, M. 2000, ApJ, 540, 489

Soker, N. \& Harpaz, A. 2000, MNRAS, 317, 861

Stobie, R.S., Kawaler, S.D., Kilkenny, D., O’Donoghue, D., \& Koen, C. 1997, MNRAS, 285, 651

Sweigart, A. \& Gross, P. 1976, ApJS, 32, 367

Sweigart, A. 1997, in The Third Conference on Faint Blue Stars, ed. A.G.D. Philip, J. Liebert, \& R.Saffer (Schenectady: L. Davis Press), 3

Thompson, M.J. et al. 1996, Science, 272, 1300 
Unno, W., Osaki, Y., Ando, Y., Saio, H., \& Shibahashi, H. 1989, Nonradial Oscillations of Stars, (Tokyo: Tokyo University Press)

van Hoolst, T., Dziembowski, W.A., \& Kawaler, S.D. 1998, MNRAS, 297, 536

Wood, P.R., Olivier, E.A., \& Kawaler, S.D. 2004, ApJ, 604, 800

Zahn, J.-P. 1992, A\&A, 265, 115 
Table 1. Parameters of the evolutionary models with $\mathrm{Z}=0.02$

\begin{tabular}{ccccc}
\hline \hline & & \multicolumn{3}{c}{$M_{\mathrm{c}, \text { flash }} / M_{\odot}$} \\
Initial Mass & $M_{\mathrm{CE}}^{\min } / M_{\odot}$ & $\begin{array}{c}\text { this work } \\
\mathrm{X}_{\text {ZAMS }}=0.74\end{array}$ & $\begin{array}{c}\text { Bono et al. 1997 } \\
\mathrm{X}_{\text {ZAMS }}=0.69\end{array}$ & $\begin{array}{c}\text { Yi et al. 2003 } \\
\mathrm{X}_{\text {ZAMS }}=0.71\end{array}$ \\
\hline $1.0 M_{\odot}$ & 0.248 & 0.4832 & 0.478 & 0.469 \\
$1.5 M_{\odot}$ & 0.269 & 0.4798 & 0.476 & 0.462 \\
$1.8 M_{\odot}$ & 0.278 & 0.4652 & 0.469 & 0.440 \\
\hline
\end{tabular}

Table 2. Representative near-ZAEHB model ${ }^{\mathrm{a}}$ pulsations, Case B

\begin{tabular}{rrrrcc}
\hline \hline Period & $\nu[\mu \mathrm{Hz}]$ & $\ell$ & $n$ & $1 / P_{\text {surf }}\left(1-C_{n, \ell}\right)$ & $\Delta \nu[\mu \mathrm{Hz}]$ \\
\hline 86.45 & 11567 & 0 & 4 & - & - \\
87.30 & 11457 & 2 & 4 & 0.086 & 17.6 \\
91.24 & 10963 & 1 & 4 & 0.087 & 7.8 \\
97.25 & 10283 & 0 & 3 & - & - \\
98.48 & 10155 & 2 & 3 & 0.084 & 19.8 \\
105.5 & 9480 & 1 & 3 & 0.086 & 8.6 \\
118.6 & 8434 & 0 & 2 & - & - \\
121.3 & 8246 & 2 & 2 & 0.081 & 25.9 \\
130.1 & 7685 & 1 & 2 & 0.087 & 2.8 \\
136.9 & 7302 & 0 & 1 & - & - \\
138.2 & 7237 & 2 & 1 & 0.084 & 11.2 \\
144.3 & 6930 & 1 & 1 & 0.087 & 2.1 \\
158.5 & 6309 & 0 & 0 & - & - \\
168.6 & 5930 & 2 & 0 & 0.059 & 91.4 \\
\hline
\end{tabular}

\footnotetext{
${ }^{\mathrm{a}} M=0.46502 M_{\odot}, M_{H}=0.00002 M_{\odot}$ $\log g=5.92, T_{\text {eff }}=32,025 \mathrm{~K}$

$v_{\text {rot }}=0.048 \mathrm{~km} / \mathrm{s}, 1 / P_{\text {surf }}=0.089 \mu \mathrm{Hz}$
} 

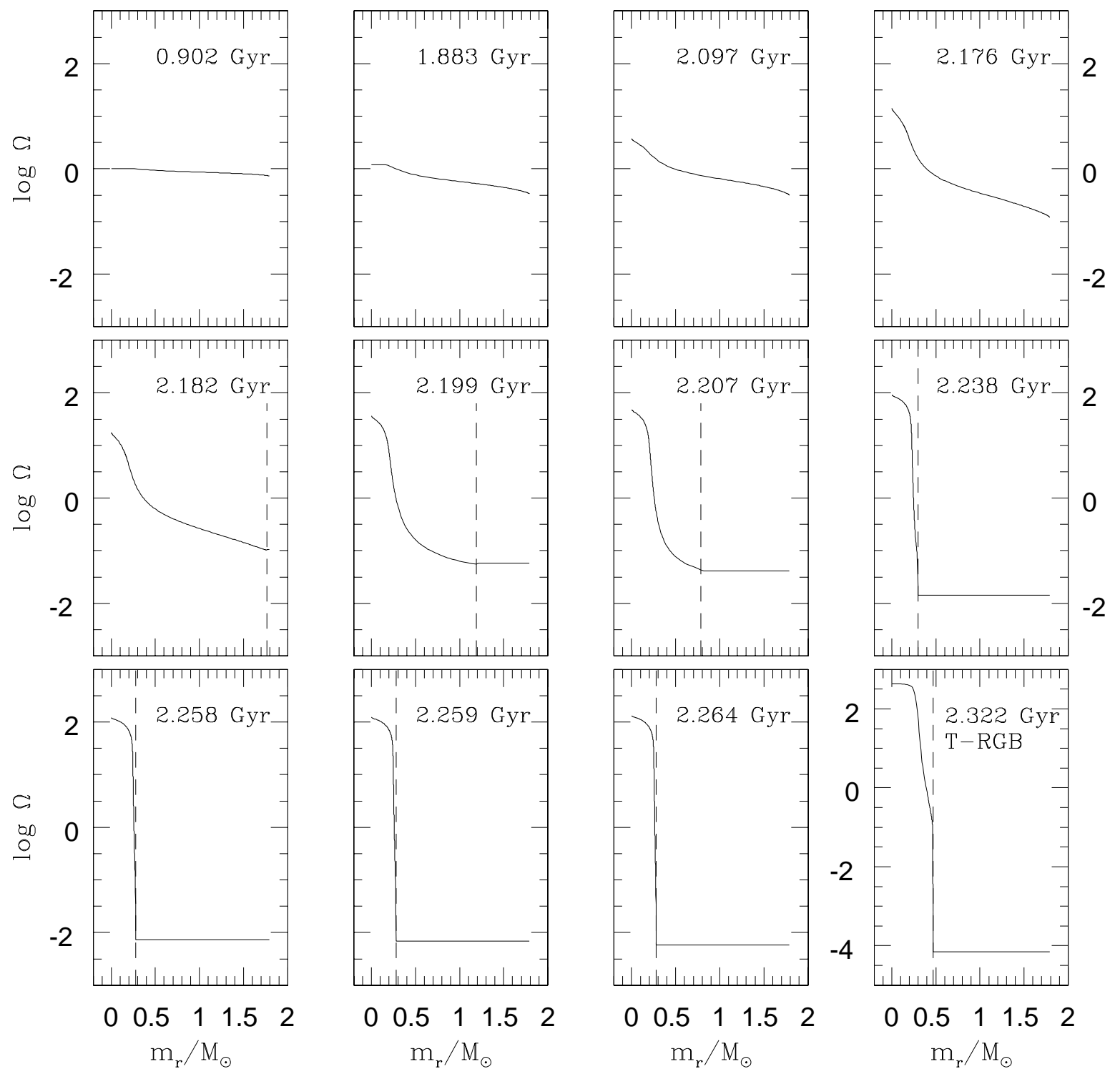

Fig. 1.- Angular velocity as a function of $m_{r}$ during early RGB evolution for the $1.8 M_{\odot}$ Case B model. The vertical dashed line denotes the base of the surface convection zone. Ages are given in each panel in Gyr. Note that the establishment of a steep angular velocity gradient occurs quite quickly as the star reaches the RGB, steepening significantly in a few times $10^{7}$ years. The maximum depth that the convection zone penetrates is in Panel 10. The last panel shows the rotation curve of the model immediately before the helium core flash. 

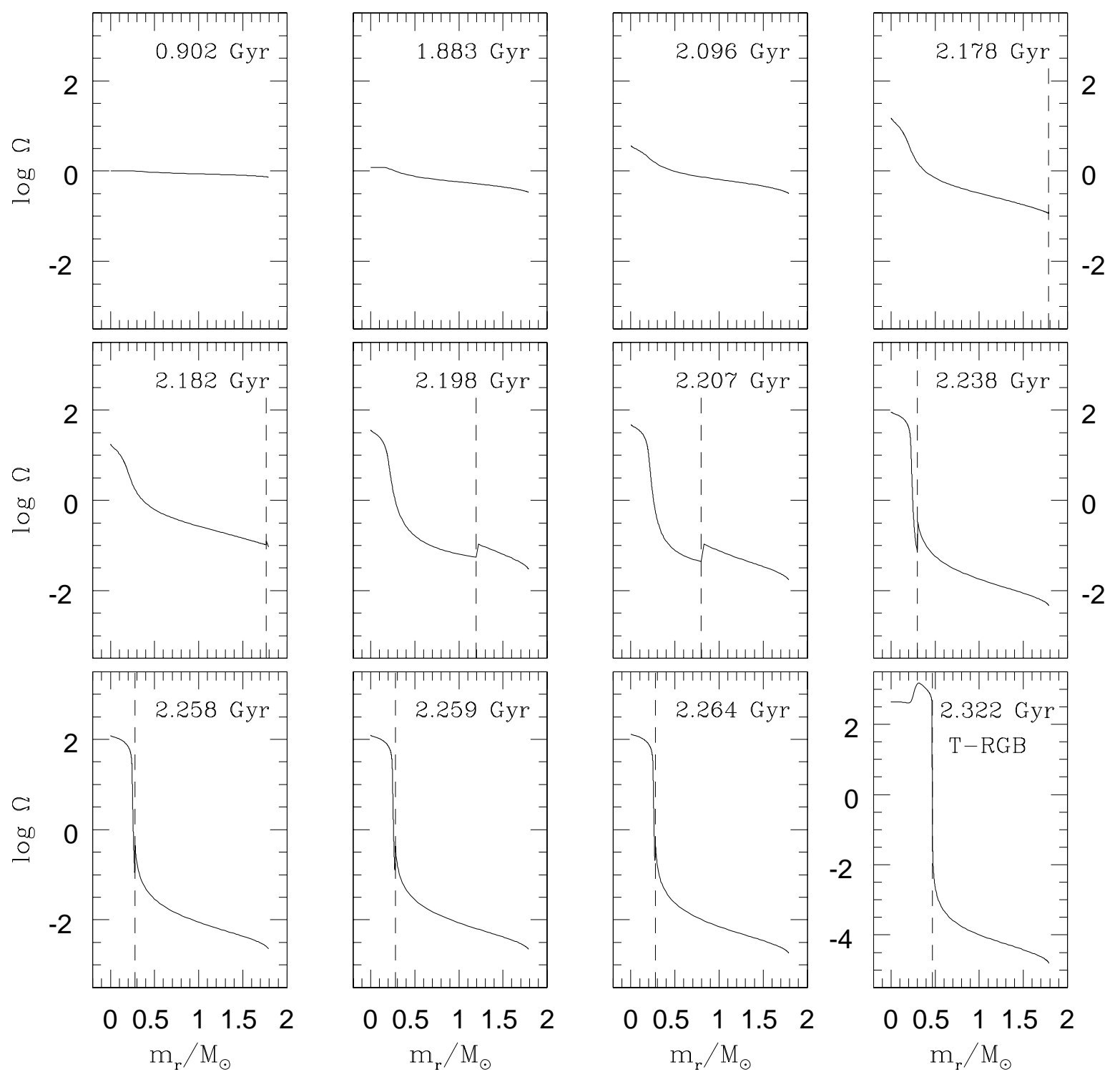

Fig. 2.- Same as Figure 1, but for Case D evolution with constant specific angular momentum in the outer convection zone. 

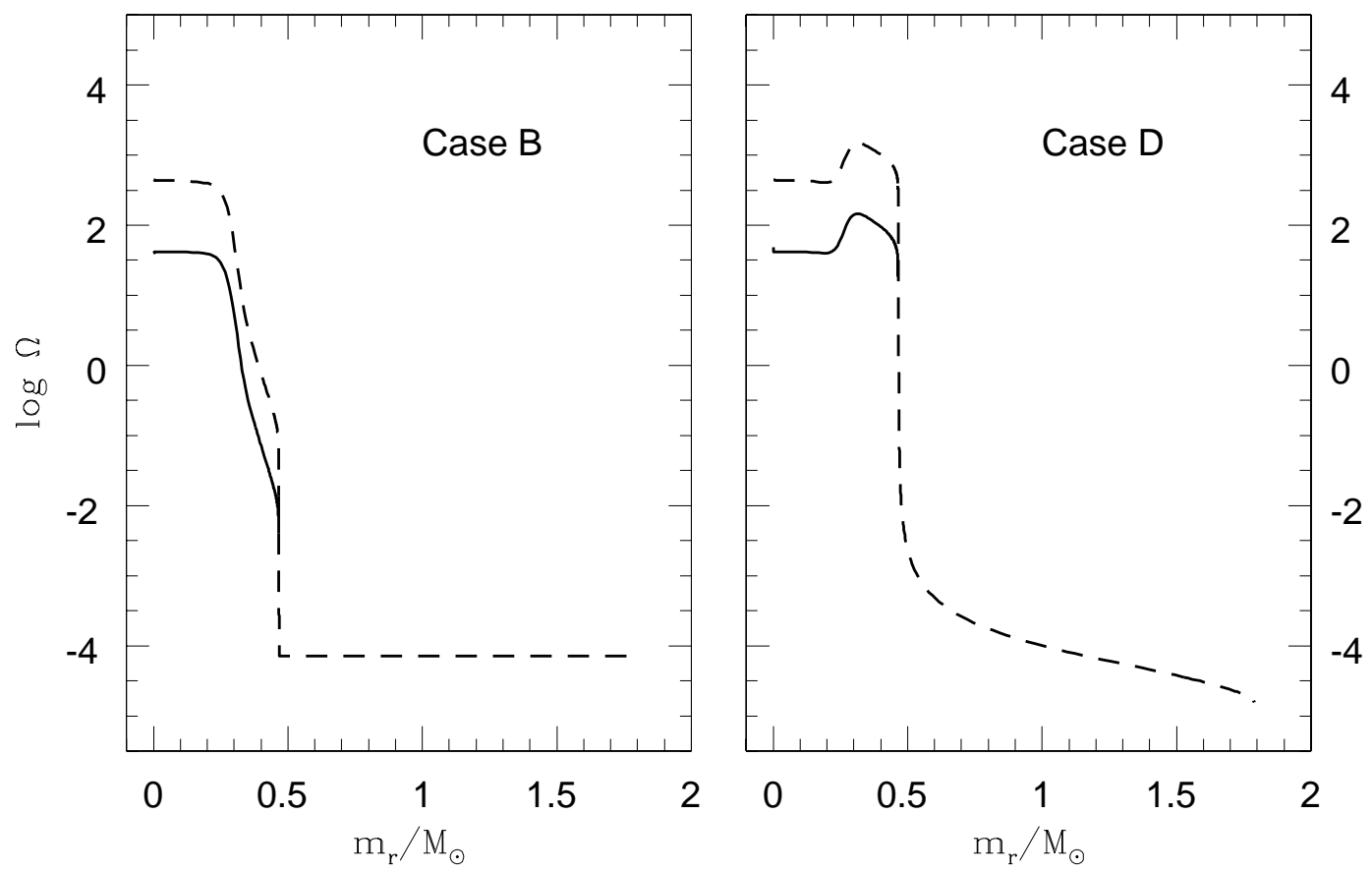

Fig. 3.- Angular velocity as a function of mass before the helium core flash (i.e. as a RGB core) and after reaching the horizontal branch (as a helium-core burning model). The dashed line represents the rotation profile on the RGB, and the solid line shows the rotation profile after reaching the HB. The two cases are Case B (left) and Case D (right). 

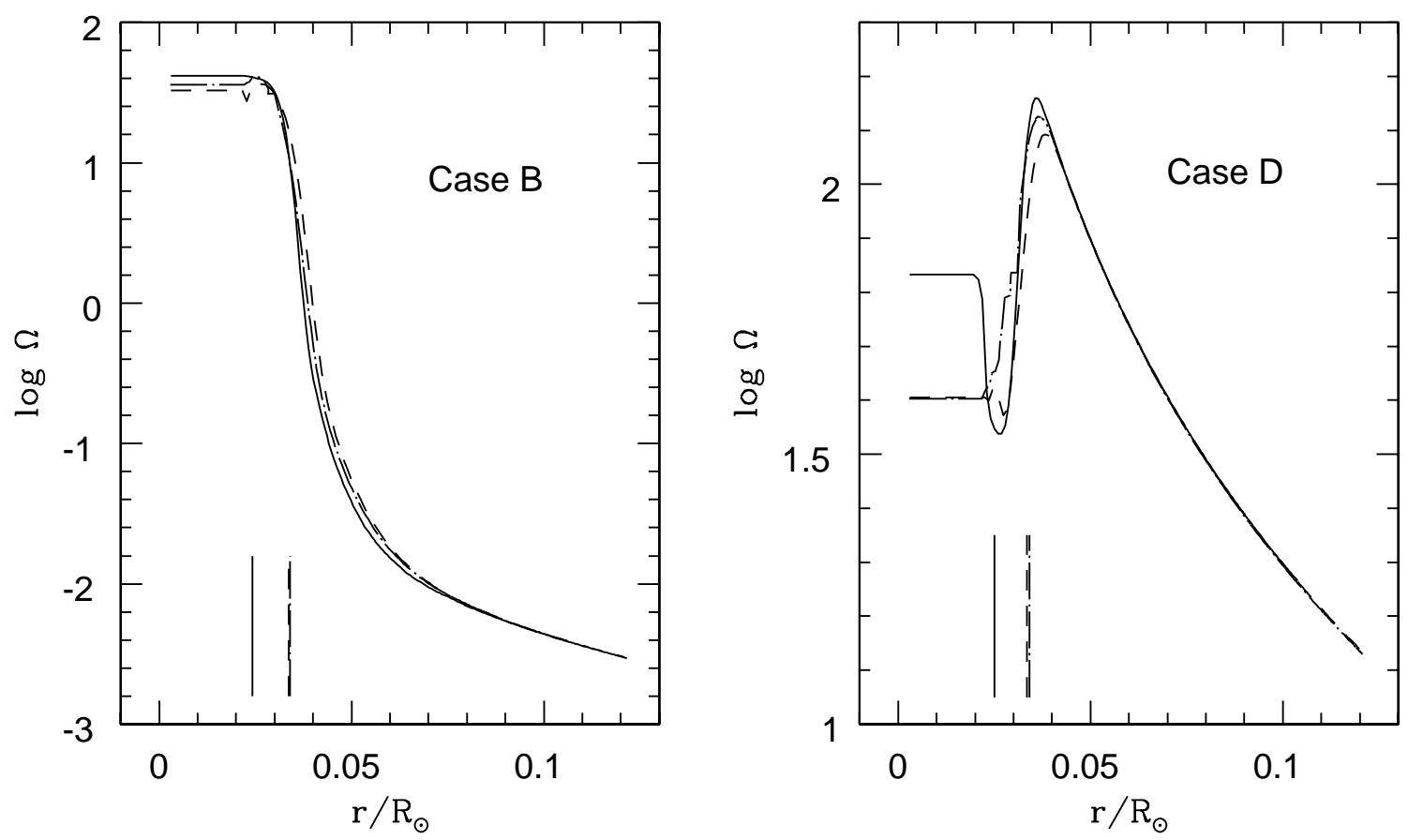

Fig. 4.- Rotation profiles for EHB models, for two cases for RGB angular momentum evolution and at different HB evolutionary stages. The left panel corresponds to Case B evolution and the right panel to Case D evolution. Models at three stages of evolution are shown, with the short vertical lines at the bottom corresponding to the transition from pure helium to the helium-depleted core. Note that the profiles show very subtle changes in the outer layers with evolution. For Case D evolution, the inner core shows changes reflecting mixing of angular momentum by the convective core as it grows and then shrinks. In Case B RGB evolution, the core spins 4 orders of magnitude faster than surface. Case D RGB evolution results in only a factor of 10 decrease in the rotation velocity from the interior to the surface of the model. 

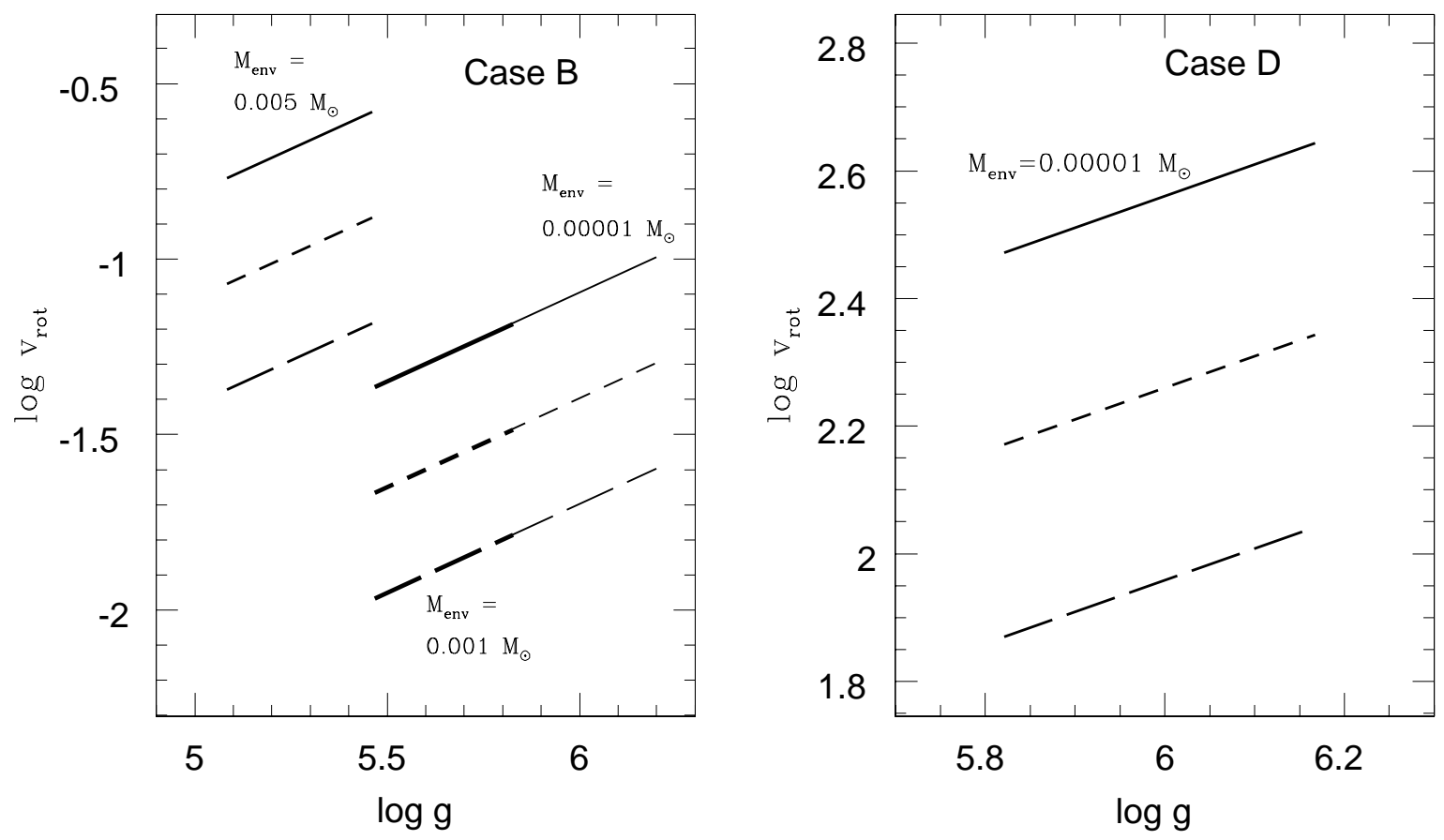

Fig. 5.- Surface rotation velocity as a function of $\log g$ for $\mathrm{sdB}$ models. The left panel shows Case B models derived from the $1.8 M_{\odot}$ sequence with surface masses as indicated, and the right panel shows representative Case D models from the same mass seed model. The solid line shows models with initial (main sequence) rotation velocities of $200 \mathrm{~km} / \mathrm{s}$; the short dashed lines had initial rotation velocities of $100 \mathrm{~km} / \mathrm{s}$, and the long dashed lines had initial rotation velocities of 50 $\mathrm{km} / \mathrm{s}$. 

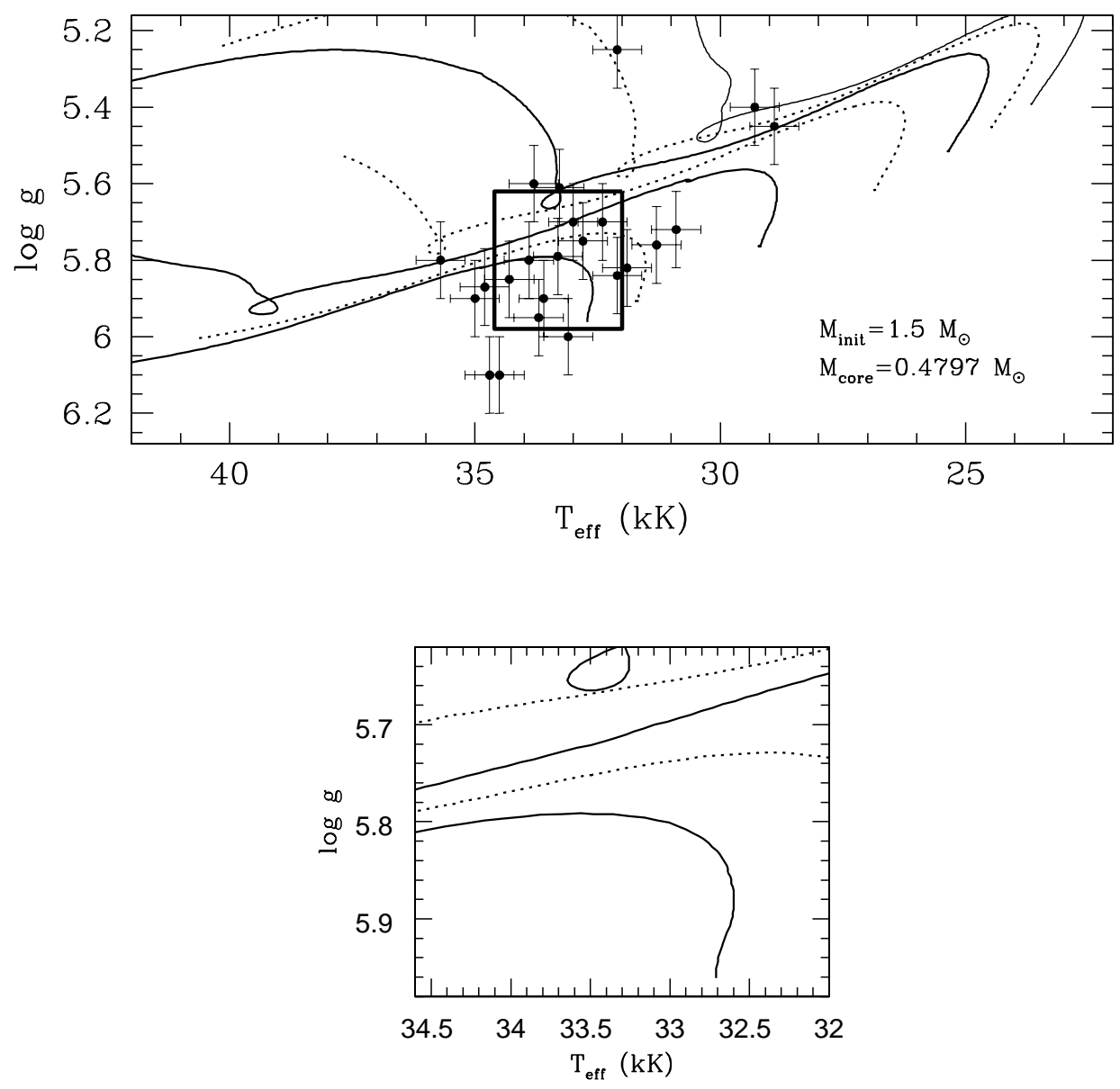

Fig. 6. - Evolutionary tracks of EHB models representative of the subdwarf B stars. Models include a core derived from the post-helium flash core of a $1.5 M_{\odot}$ star with increasing hydrogenrich envelopes with increasing $T_{\text {eff }}$. Alternating tracks can be distinguished by the different line types. Envelope masses are 0.00001, 0.00004, 0.00032, 0.00122, 0.00232, 0.00322, 0.00422 $M_{\odot}$, running from lower left to upper right. Data points are the pulsating sdB stars (from Kilkenny 2001), The box is centered at the mean position of the sdB pulsators, and the size of dimensions of the box are proportional to the width of the distributions. The lower plot is an enlargement of the box, showing that sdB models of various evolutionary stages underly the positions of the pulsating sdB stars. 

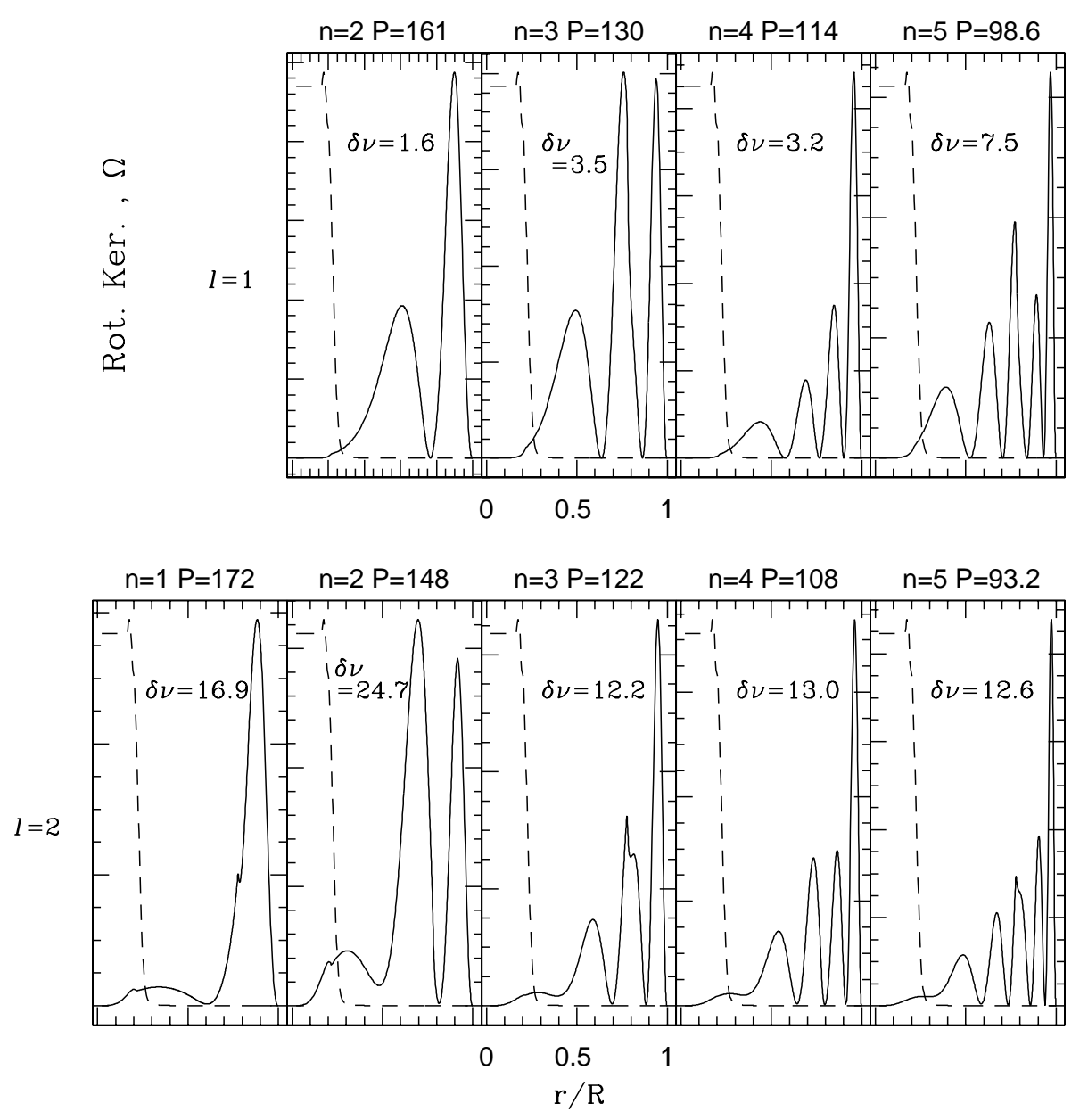

Fig. 7.- Ssample rotation kernels for a representative nearing the end of core helium burning, for modes with periods and degrees as indicated. Dashed lines represent $\log \Omega(r)$ for Case B angular momentum transport in the RGB convection zone. Splitting values are in $\mu \mathrm{Hz}$. 
Table 3. Representative near-ZAEHB model ${ }^{\mathrm{b}}$ pulsations, Case D.

\begin{tabular}{rrrrcc}
\hline \hline Period & $\nu[\mu \mathrm{Hz}]$ & $\ell$ & $n$ & $1 / P_{\text {surf }}\left(1-C_{n, \ell}\right)$ & $\Delta \nu[\mu \mathrm{Hz}]$ \\
\hline 84.73 & 11800 & 0 & 4 & - & - \\
85.50 & 11699 & 2 & 4 & 18.0 & 29.3 \\
89.30 & 11198 & 1 & 4 & 18.1 & 32.2 \\
95.88 & 10430 & 0 & 3 & - & - \\
97.22 & 10287 & 2 & 3 & 17.6 & 32.0 \\
104.5 & 9574 & 1 & 3 & 17.9 & 39.8 \\
117.1 & 8540 & 0 & 2 & - & - \\
119.5 & 8372 & 2 & 2 & 17.1 & 30.2 \\
126.9 & 7880 & 1 & 2 & 18.1 & 28.9 \\
133.3 & 7504 & 0 & 1 & - & - \\
134.9 & 7414 & 2 & 1 & 17.4 & 25.0 \\
142.4 & 7023 & 1 & 1 & 18.1 & 34.8 \\
157.6 & 6344 & 0 & 0 & - & - \\
167.9 & 5956 & 2 & 0 & 12.3 & 47.3 \\
\hline
\end{tabular}

$$
\begin{aligned}
& \mathrm{b} M=0.46413 M_{\odot}, M_{H}=0.00002 M_{\odot} \\
& \log g=5.93, T_{\text {eff }}=32,114 \mathrm{~K} \\
& v_{\text {rot }}=10 \mathrm{~km} / \mathrm{s}, 1 / P_{\text {surf }}=18.6 \mu \mathrm{Hz}, J / J_{\text {init }}=0.045
\end{aligned}
$$


Table 4. Representative late-ZAEHB model ${ }^{\mathrm{c}}$ pulsations, Case B

\begin{tabular}{cccccc}
\hline \hline Period & $\nu[\mu \mathrm{Hz}]$ & $\ell$ & $n$ & $1 / P_{\text {surf }}\left(1-C_{n, \ell}\right)$ & $\Delta \nu[\mu \mathrm{Hz}]$ \\
\hline 82.37 & 12140 & 0 & 6 & - & - \\
84.20 & 11876 & 2 & 6 & 0.051 & 13.6 \\
87.85 & 11384 & 1 & 6 & 0.052 & 7.1 \\
91.14 & 10972 & 0 & 5 & - & - \\
93.21 & 10728 & 2 & 5 & 0.051 & 12.6 \\
98.65 & 10138 & 1 & 5 & 0.052 & 7.5 \\
105.6 & 9467 & 0 & 4 & - & - \\
108.3 & 9238 & 2 & 4 & 0.050 & 13.0 \\
113.9 & 8784 & 1 & 4 & 0.052 & 3.2 \\
118.8 & 8417 & 0 & 3 & - & - \\
121.9 & 8206 & 2 & 3 & 0.050 & 12.2 \\
130.3 & 7677 & 1 & 3 & 0.052 & 3.5 \\
141.8 & 7052 & 0 & 2 & - & - \\
147.6 & 6774 & 2 & 2 & 0.046 & 24.7 \\
161.4 & 6196 & 1 & 2 & 0.052 & 1.6 \\
167.2 & 5980 & 0 & 1 & - & - \\
171.5 & 5832 & 2 & 1 & 0.049 & 16.9 \\
179.4 & 5573 & 1 & 1 & 0.052 & 0.9 \\
185.9 & 5379 & 0 & 0 & - & - \\
191.1 & 5232 & 2 & 0 & 0.044 & 75.9 \\
\hline
\end{tabular}

${ }^{\mathrm{c}} M=0.47991 M_{\odot}, M_{H}=0.00001 M_{\odot}$ $\log g=5.80, T_{\text {eff }}=33,113 \mathrm{~K}$

$v_{\text {rot }}=0.034 \mathrm{~km} / \mathrm{s}, 1 / P_{\text {surf }}=0.0532 \mu \mathrm{Hz}$ 
Table 5. Representative late-ZAEHB model ${ }^{\mathrm{d}}$ pulsations, Case D

\begin{tabular}{rrrrcc}
\hline \hline Period & $\nu[\mu \mathrm{Hz}]$ & $\ell$ & $n$ & $1 / P_{\text {surf }}\left(1-C_{n, \ell}\right)$ & $\Delta \nu[\mu \mathrm{Hz}]$ \\
\hline 82.65 & 12099 & 0 & 6 & - & - \\
83.33 & 11999 & 2 & 6 & 15.3 & 31.0 \\
86.89 & 11508 & 1 & 6 & 15.5 & 31.5 \\
91.45 & 12099 & 0 & 5 & - & - \\
92.26 & 10839 & 2 & 5 & 15.3 & 30.2 \\
97.68 & 10239 & 1 & 5 & 15.4 & 38.7 \\
106.0 & 9435 & 0 & 4 & - & - \\
107.2 & 9331 & 2 & 4 & 15.1 & 29.1 \\
112.6 & 8881 & 1 & 4 & 15.5 & 29.5 \\
119.1 & 8390 & 0 & 3 & - & - \\
120.6 & 8287 & 2 & 3 & 14.9 & 27.3 \\
129.0 & 7753 & 1 & 3 & 15.5 & 36.5 \\
142.3 & 7026 & 0 & 2 & - & - \\
146.5 & 6828 & 2 & 2 & 13.9 & 31.4 \\
159.7 & 6261 & 1 & 2 & 15.5 & 30.4 \\
167.8 & 5961 & 0 & 1 & - & - \\
169.8 & 5890 & 2 & 1 & 14.6 & 23.6 \\
177.5 & 5633 & 1 & 1 & 15.5 & 27.3 \\
186.6 & 5360 & 0 & 0 & - & - \\
190.7 & 5243 & 2 & 0 & 12.7 & 52.0 \\
\hline
\end{tabular}

$$
\begin{aligned}
& { }^{\mathrm{d}} M=0.47979 M_{\odot}, M_{H}=0.00001 M_{\odot} \\
& \log g=5.81, T_{\text {eff }}=32,945 \mathrm{~K} \\
& v_{\text {rot }}=10 \mathrm{~km} / \mathrm{s}, 1 / P_{\text {surf }}=15.8 \mu \mathrm{Hz}, J / J_{\text {init }}=0.067
\end{aligned}
$$


Table 6. Representative post-ZAEHB model ${ }^{\mathrm{e}}$ pulsations, Case B

\begin{tabular}{cccccc}
\hline \hline Period & $\nu[\mu \mathrm{Hz}]$ & $\ell$ & $n$ & $1 / P_{\text {surf }}\left(1-C_{n, \ell}\right)$ & $\Delta \nu[\mu \mathrm{Hz}]$ \\
\hline 83.49 & 11977 & 0 & 7 & - & - \\
83.75 & 11934 & 2 & 7 & 0.042 & 9.3 \\
87.29 & 11453 & 1 & 7 & 0.043 & 5.7 \\
93.48 & 10697 & 0 & 6 & - & - \\
94.09 & 10626 & 2 & 6 & 0.042 & 11.6 \\
97.43 & 10258 & 1 & 6 & 0.043 & 2.4 \\
102.9 & 9716 & 0 & 5 & - & - \\
104.8 & 9545 & 2 & 5 & 0.040 & 23.6 \\
111.7 & 9418 & 1 & 5 & 0.043 & 2.0 \\
115.7 & 8642 & 0 & 4 & - & - \\
116.7 & 8567 & 2 & 4 & 0.041 & 17.8 \\
125.9 & 7943 & 1 & 4 & 0.043 & 3.0 \\
134.5 & 7438 & 0 & 3 & - & - \\
135.8 & 7364 & 2 & 3 & 0.039 & 38.6 \\
142.0 & 7041 & 1 & 3 & 0.043 & 0.9 \\
147.0 & 6804 & 0 & 2 & - & - \\
147.4 & 6784 & 2 & 2 & 0.038 & 103 \\
173.5 & 5765 & 2 & 1 & 0.043 & 17.7 \\
174.9 & 5716 & 1 & 2 & 0.043 & 0.2 \\
176.3 & 5673 & 0 & 1 & - & - \\
192.8 & 5188 & 2 & 0 & 0.034 & 210 \\
\hline
\end{tabular}

${ }^{\mathrm{e}} M=0.48020 M_{\odot}, M_{H}=0.00042 M_{\odot}$ $\log g=5.72, T_{\text {eff }}=33,334 \mathrm{~K}$

$v_{\text {rot }}=0.030 \mathrm{~km} / \mathrm{s}, 1 / P_{\text {surf }}=0.043 \mu \mathrm{Hz}$ 
Table 7. Representative post-EHB model ${ }^{\mathrm{f}}$ pulsations, Case D

\begin{tabular}{cccccc}
\hline \hline Period & $\nu[\mu \mathrm{Hz}]$ & $\ell$ & $n$ & $1 / P_{\text {surf }}\left(1-C_{n, \ell}\right)$ & $\Delta \nu[\mu \mathrm{Hz}]$ \\
\hline 85.23 & 11800 & 0 & 7 & - & - \\
85.46 & 11701 & 2 & 7 & 13.8 & 28.4 \\
88.48 & 11302 & 1 & 7 & 13.9 & 39.6 \\
94.79 & 10257 & 0 & 6 & - & - \\
95.73 & 10444 & 2 & 6 & 13.5 & 33.0 \\
99.48 & 10052 & 1 & 6 & 14.1 & 30.7 \\
104.0 & 9615 & 0 & 5 & - & - \\
105.7 & 9463 & 2 & 5 & 12.9 & 38.6 \\
113.8 & 8791 & 1 & 5 & 14.0 & 36.5 \\
118.1 & 8467 & 0 & 4 & - & - \\
118.9 & 8414 & 2 & 4 & 13.3 & 28.6 \\
126.7 & 7895 & 1 & 4 & 13.9 & 46.1 \\
135.8 & 7364 & 0 & 3 & - & - \\
137.8 & 7257 & 2 & 3 & 12.4 & 49.7 \\
144.9 & 6902 & 1 & 3 & 14.0 & 26.3 \\
148.0 & 6758 & 2 & 2 & 12.9 & 64.5 \\
148.2 & 6748 & 0 & 2 & - & - \\
177.5 & 5634 & 2 & 1 & 14.1 & 30.3 \\
179.3 & 5577 & 1 & 2 & 14.0 & 21.1 \\
180.7 & 5534 & 0 & 1 & - & - \\
194.0 & 5154 & 2 & 0 & 11.3 & 137.7 \\
\hline
\end{tabular}

${ }^{\mathrm{f}} M=0.48010 M_{\odot}, M_{H}=0.00047 M_{\odot}$ $\log g=5.70, T_{\text {eff }}=33,116 \mathrm{~K}$

$v_{\text {rot }}=10 \mathrm{~km} / \mathrm{s}, 1 / P_{\text {surf }}=14.1 \mu \mathrm{Hz}, J / J_{\text {init }}=0.076$ 